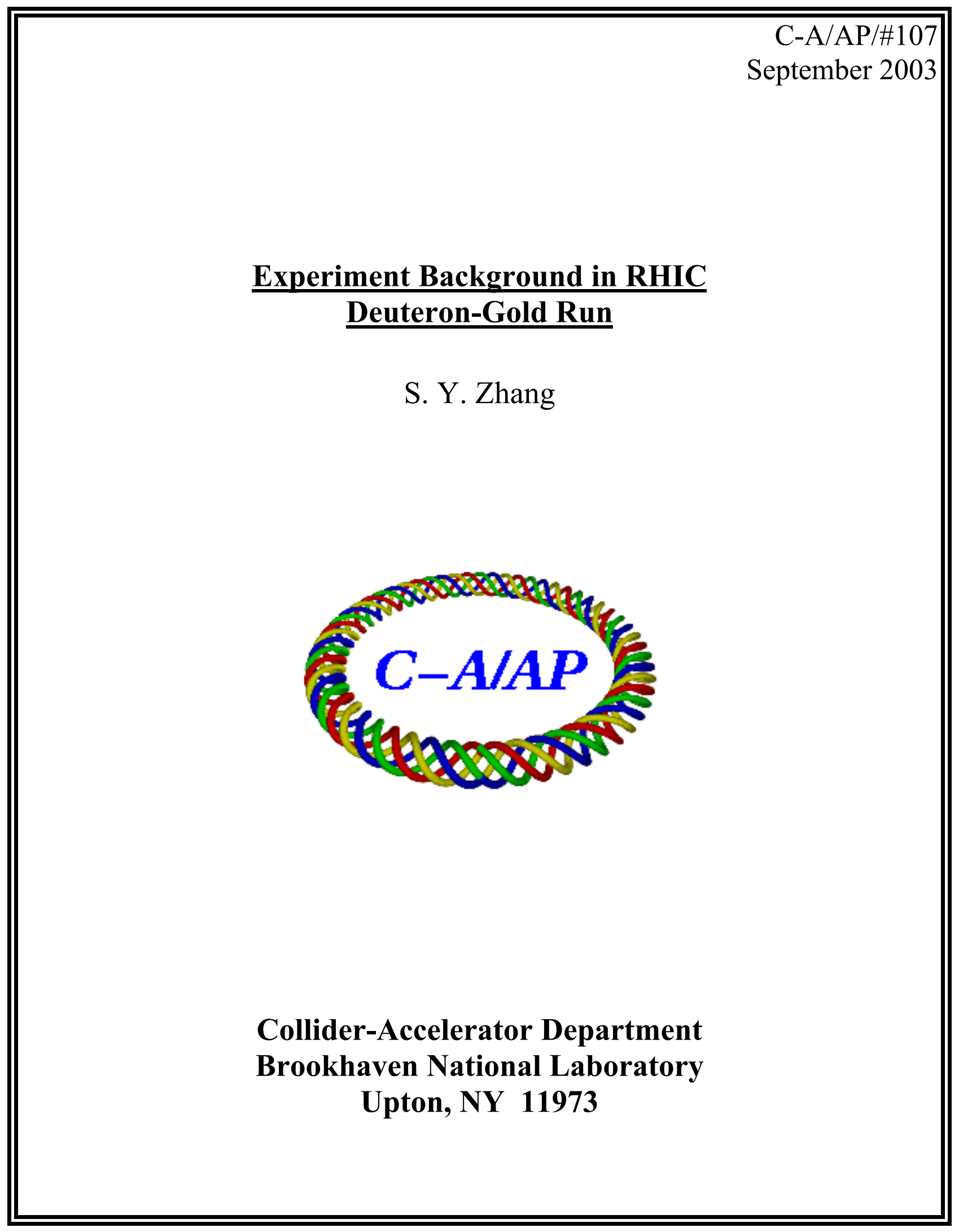




\title{
Experiment Background in RHIC Deuteron-Gold Run
}

\author{
S.Y. Zhang \\ I. Introduction
}

In RHIC 2003 deuteron-gold (d-Au) run, high experiment background had raised concern, which requires understanding in view of planning of 2003-2004 gold-gold ( $\mathrm{Au}-\mathrm{Au})$ run and future heavy ion operations.

In section II of this study report, 'conventions' will be proposed, which include the relevant machine and beam parameter, vacuum condition, beam intensity, cross sections such as the nuclear collision, Coulomb dissociation, and beam-gas collision. From these conventions, the ZDC (Zero Degree Calorimeter) coincident rate, singles rate, and beam-gas collision rate, etc. can be derived. For not-yet-settled issues, such as the d-Au hadronic interaction and gold beam gas interaction, the convention is defined according to the measurements and observation.

In section III, typical background cases will be analyzed to identify the beam-beam and beamgas contributions to background.

In section IV, the 2003 deuteron-gold run background issues will be reviewed based on the experiment statistics and several operational events during the d-Au run. These events are (1) switch optics dAu2 to dAu3, (2) eliminating beam coherence, (3) 110-bunch changed to 56-bunch mode, and (4) switch optics dAu3 to dAu4.

A discussion of the background problem with respect to each experiment will be presented in section V, and the beam-gas factor in background will be discussed in section VI.

Finally, a summary of findings and a brief discussion for next gold-gold run will be presented.

\section{Convention and parameter}

Following conventions are proposed to develop typical background and coincident rates, which are then compared with the observation and measurements in the past Au-Au run of 2001, and d-Au run of 2003. Further examination is needed for verification and modification.

1. Throughout this report, background is defined as the ZDC singles. In specific, it is the average of ZDC singles in Au-direction and d-direction [1]. ZDCcoin is defined as the ZDC coincident rate. Most critical criterion for all experiments is the ratio of background/ ZDCcoin. Experience shows that for d-Au run, good background/ZDCcoin ratio was around 5 , and the ratio of 15 was considered high. Note that according to this definition, ZDCcoin is a part of the background rate. Also, it was observed that for high background, say $1 \mathrm{MHz}$ or higher, ZDCcoin was contaminated, i.e. larger than that should be.

2. Beam intensities for 56-bunch mode are assumed as follows. Total gold beam intensity is $\mathrm{N}_{\mathrm{Au}}=40 \times 10^{9}$ ions, i.e., bunch intensity is $\mathrm{N}_{\mathrm{Au}, \mathrm{bh}}=0.7 \times 10^{9}$ ions, and total deuteron beam intensity is $\mathrm{N}_{\mathrm{d}}=56 \times 10^{11}$ ions, with bunch intensity of $\mathrm{N}_{\mathrm{d}, \mathrm{bh}}=1 \times 10^{11}$ ions. 
3. Normalized beam emittance at collision is $\varepsilon_{\mathrm{N}}=15 \pi \mu \mathrm{m}$, and bunch length at collision is $\ell=$ $1.5 \mathrm{~m}$. The beam radius at $\beta^{*}=2 \mathrm{~m}$ is therefore $\mathrm{a}=\sqrt{\varepsilon_{\mathrm{N}} \beta^{*} /(\beta c)}=0.53 \mathrm{~mm}$. Assuming that particles are uniformly distributed in the cylinder with radius of $2 \sigma=2 \mathrm{a} / \sqrt{6}=0.43 \mathrm{~mm}$ and length of $1.5 \mathrm{~m}$, the d-ion density in a bunch is $\mathrm{D}_{\mathrm{d}}=1.15 \times 10^{17} \mathrm{~m}^{-3}$ for $\mathrm{N}_{\mathrm{d}, \mathrm{bh}}=1 \times 10^{11}$ ions, and Au-ion density is $\mathrm{D}_{\mathrm{Au}}=8.05 \times 10^{14} \mathrm{~m}^{-3}$ for $\mathrm{N}_{\mathrm{Au}, \mathrm{bh}}=0.7 \times 10^{9}$ ions.

4. For vacuum parameters, assume the effective length of IR to be $\ell_{\text {vac }}=14 \mathrm{~m}$. Assume that the gas consists equally of $\mathrm{H}_{2}$ and $\mathrm{CO}$, then the average mass of target molecule is $A_{t}=15$. Since the gauges are located at $7.6 \mathrm{~m}$ from the IP, ratio of average pressure vs. gauge readings is assumed to be $\mathrm{P}_{\text {ave. }} / \mathrm{P}=10$. This ratio represents the pipe conductance effect. As a reference, for a typical IR, it was calculated that this ratio is 8.5 for $\mathrm{H}_{2}$, and 28 for $\mathrm{CO}$ [2].

5. Nuclear collision cross sections are calculated using 'billiard ball' model, which is

$$
\sigma_{\mathrm{N}}=\pi\left(1.2 \times 10^{-15} \times\left(\mathrm{A}_{\mathrm{p}}^{1 / 3}+\mathrm{A}_{\mathrm{t}}^{1 / 3}\right)\right)^{2} \mathrm{~m}^{2}
$$

where $A_{p}$ and $A_{t}$ are the masses of projectile and target, respectively. This way, gold-gas cross section is calculated as $\sigma_{\text {Au-gas }}=3.1 \mathrm{~b}$, deuteron-gas cross section is $\sigma_{\text {d-gas }}=0.63 \mathrm{~b}$, where $1 \mathrm{~b}=1 \mathrm{barn}=10^{-28} \mathrm{~m}^{-2}$. Also, Au-Au nuclear collision cross section is $6.1 \mathrm{~b}$. which is close to 'geometrical' cross section of $7 \mathrm{~b}[3,4]$. The $\mathrm{d}$-Au nuclear collision cross section is $\sigma_{\mathrm{d}-\mathrm{Au}}=2.3 \mathrm{~b}$.

6. For Au-Au run, the total cross section responsible to the ZDCcoin is about $\sigma_{\mathrm{Au}-\mathrm{Au}}=6.1+4$ $\approx 10 \mathrm{~b}$, where $4 \mathrm{~b}$ is the mutual Coulomb dissociation cross section. Au-Au single Coulomb dissociation cross section is $\sigma_{\text {singlec,Au-Au }}=92 \mathrm{~b}[3,4]$, which contributes to singles.

7. The mechanism of d-Au hadronic interaction is not very clear at the moment. It is agreed that the $\mathrm{d}-\mathrm{Au}$ (dir) cross section is $\sigma_{\mathrm{d}-\mathrm{Au}(\mathrm{dir})}=\sigma_{\mathrm{d}-\mathrm{Au}}=2.3 \mathrm{~b}$, and the Au-d(dir) cross section is measured to be about $20 \%$ of $\sigma_{\mathrm{d}-\mathrm{Au}(\text { (dir) }}$ [5], and therefore $\sigma_{\text {Au-d(dir) }}=0.46 \mathrm{~b}$. The d-Au(dir) single Coulomb dissociation cross section is negligible, whereas $\sigma_{\text {singleC,Au-d(dir) }}=1.25 \mathrm{~b}$ [6]. This way the cross section responsible for $\mathrm{d}-\mathrm{Au}$ ZDCcoin is $0.46 \mathrm{~b}$. The cross section responsible for Au direction singles is $2.3 \mathrm{~b}$, and for d-direction is $0.46+1.25=1.71 \mathrm{~b}$. The verification of these parameters is not straightforward, and therefore it is an undergoing effort.

8. ZDCcoin is calculated by letting the total ions in one beam to collide with another beam, which is represented by the ions of one bunch in the cylinder with the length of $1.5 \mathrm{~m}$ and the radius of $0.43 \mathrm{~mm}$. Let the machine circumference be $\mathrm{C}$, and velocity of light be $\mathrm{c}$. Then the $\mathrm{Au}-\mathrm{Au}$ coincident rate is

$$
\mathrm{r}_{\mathrm{Au}-\mathrm{Au}}=\mathrm{N}_{\mathrm{Au}} \mathrm{D}_{\mathrm{Au}} \sigma_{\mathrm{Au}-\mathrm{Au}} \mathrm{c} \ell / \mathrm{C}
$$

Using $\mathrm{N}_{\mathrm{Au}}=40 \times 10^{9}, \mathrm{D}_{\mathrm{Au}}=8.05 \times 10^{14} \mathrm{~m}^{-3}, \sigma_{\mathrm{Au}-\mathrm{Au}}=10 \mathrm{~b}=10 \times 10^{-28} \mathrm{~m}^{-2}$, and $\ell=1.5 \mathrm{~m}$, we get $\mathrm{r}_{\mathrm{Au}-\mathrm{Au}}=3.8 \mathrm{kHz}$, which is close to the observed ones. For example, the peak ZDCcoin of Phenix in the Fill 1812 of 2001 run was $3.7 \mathrm{kHz}$ [7], where the beam intensity was $30 \times 10^{9}$ rather than $40 \times 10^{9}$ ions (Convention 2), but $\beta^{*}=1 \mathrm{~m}$. The $\mathrm{d}-\mathrm{Au}$ ZDCcoin is calculated in the same way to result in $\mathrm{r}_{\mathrm{d}-\mathrm{Au}}=25 \mathrm{kHz}$, which is also close to the observed ZDCcoin. For example, the peak ZDCcoin of Phenix in Fill 3154 of d-Au run was $26 \mathrm{kHz}$, 
where the total deuteron beam intensity was $60 \times 10^{11}$ ions, and the total gold beam intensity was $25 \times 10^{9}$ ions. Error here is considered acceptable.

9. For $\mathrm{Au}-\mathrm{Au}$ run, the single Coulomb dissociation is assumed to be the dominant mechanism in beam-beam created singles, then the background rate is

$$
\mathrm{Bk}_{\mathrm{Au}-\mathrm{Au}}=\mathrm{N}_{\mathrm{Au}} \mathrm{D}_{\mathrm{Au}}\left(\sigma_{\text {singlec,Au-Au }}+\sigma_{\mathrm{Au}-\mathrm{Au}}\right) \mathrm{c} \ell / \mathrm{C}
$$

substituting $\sigma_{\text {singlec,Au-Au }}=92 \mathrm{~b}$ and $\sigma_{\mathrm{Au}-\mathrm{Au}}=10 \mathrm{~b}$ results in $\mathrm{Bk}_{\mathrm{Au}-\mathrm{Au}}=38.5 \mathrm{kHz}$.

10. For d-Au run, the beam-beam created background in Au-direction is calculated using the cross section of $2.3 \mathrm{~b}$, which results in $\mathrm{Bk}_{\mathrm{d}-\mathrm{Au}(\mathrm{dir})}=124 \mathrm{kHz}$. The singles on the d-direction is calculated using the cross section of $1.71 \mathrm{~b}$, which results in $\mathrm{Bk}_{\text {Au-d(dir) }}=90 \mathrm{kHz}$. The background, which is the average of singles, therefore is $\mathrm{Bk}_{\mathrm{d}-\mathrm{Au}}=107 \mathrm{kHz}$.

11. Beam-gas nuclear collision rate is calculated as follows. The gas molecules' density is

$$
\mathrm{D}_{\text {gas }}=3.3 \times 10^{22} \mathrm{Pm}^{-3}
$$

where $\mathrm{P}$ is the pressure in Torr. The background rate caused by gold beam colliding with gas molecules is

$$
\mathrm{Bk}_{\text {Au-gas }}=\mathrm{N}_{\mathrm{Au}} \mathrm{D}_{\text {gas }}\left(\mathrm{P}_{\text {ave }} / \mathrm{P}\right) \sigma_{\text {Au-gas }} \mathrm{c} \ell_{\text {vac }} / \mathrm{C}
$$

For $\mathrm{P}=10^{-9}$ Torr, using $\mathrm{P}_{\text {ave }} / \mathrm{P}=10, \ell_{\mathrm{vac}}=14 \mathrm{~m}$, one gets $\mathrm{Bk}_{\text {Au-gas }}=4.5 \mathrm{kHz}$. This rate is significantly lower than the observed one in $2001 \mathrm{Au}-\mathrm{Au}$ run, which was more like $20 \mathrm{kHz}$ to $30 \mathrm{kHz}$. It looks that for Au-gas collision, other mechanism than the nuclear collision might be involved. For instance, the $\mathrm{Z}^{2}$ effect enhanced ionization may contribute to the ZDC rate. We tentatively modify the Au-gas collision cross section by factor of 6 , from $3.1 \mathrm{~b}$ to $18.6 \mathrm{~b}$, which leads to $\mathrm{Bk}_{\text {Au-gas }}=27 \mathrm{kHz}$. For d-gas nuclear collision, using the cross section of 0.63 $\mathrm{b}$, for $\mathrm{P}=10^{-9}$ Torr, one gets $\mathrm{Bk}_{\mathrm{d}-\mathrm{gas}}=128 \mathrm{kHz}$, which is approximately agreeable to the observation. Beam-gas background for $\mathrm{P}=10^{-9}$ Torr, therefore, is $(27+128) / 2=78 \mathrm{kHz}$.

12. Luminosity is calculated from the ZDCcoin by

$$
\mathrm{L}_{\mathrm{bb}}=\mathrm{ZDCcoin} / \sigma
$$

For $\mathrm{Au}-\mathrm{Au}$ run, we have $\mathrm{L}_{\mathrm{bb}}=3.8 \times 10^{3} / 10 \times 10^{-28}=3.8 \times 10^{30} \mathrm{~m}^{-2} \mathrm{~s}^{-1}=3.8 \times 10^{26} \mathrm{~cm}^{-2} \mathrm{~s}^{-1}$. In Fill 1812 of $2001 \mathrm{Au}-\mathrm{Au}$ run, the peak luminosity at Phenix was $\mathrm{L}_{\mathrm{bb}}=3.7 \times 10^{26} \mathrm{~cm}^{-2} \mathrm{~s}^{-1}$.

For d-Au run, the luminosity from (6) is $\mathrm{L}_{\mathrm{bb}}=25 \times 10^{3} / 0.46 \times 10^{-28}=5.4 \times 10^{28} \mathrm{~cm}^{-2} \mathrm{~s}^{-1}$, and the peak luminosity in $2003 \mathrm{~d}-\mathrm{Au}$ run at Phenix was $\mathrm{L}_{\mathrm{bb}}=5.1 \times 10^{28} \mathrm{c} \mathrm{m}^{-2} \mathrm{~s}^{-1}[8]$.

In summary, the ZDC coincident rate, the beam-beam background, the beam-gas background, and the responsible cross sections in d-Au run are shown in Table 1.

\begin{tabular}{|c|c|c|c|}
\hline & ZDCcoin & Background & Background \\
\hline & & beam-beam & beam-gas, P = 1e-9 Torr \\
\hline $\mathrm{Au}-\mathrm{Au}$ & $3.8 \mathrm{kHz} / 10 \mathrm{~b}$ & $38.5 \mathrm{kHz} / 102 \mathrm{~b}$ & $27 \mathrm{kHz} / 18.6 \mathrm{~b}$ \\
\hline $\mathrm{d}-\mathrm{Au}$, total & $25 \mathrm{kHz} / 0.46 \mathrm{~b}$ & $107 \mathrm{kHz}$ & $78 \mathrm{kHz}$ \\
\hline $\mathrm{d}-\mathrm{Au}$, Au (dir) & & $124 \mathrm{kHz} / 2.3 \mathrm{~b}$ & $27 \mathrm{kHz} / 18.6 \mathrm{~b}$ \\
\hline $\mathrm{d}-\mathrm{Au}, d$ (dir) & & $90 \mathrm{kHz} / 1.71 \mathrm{~b}$ & $128 \mathrm{kHz} / 0.63 \mathrm{~b}$ \\
\hline
\end{tabular}

Table 1 


\section{Beam-gas and beam-beam background}

\section{Beam-gas and beam-beam created background}

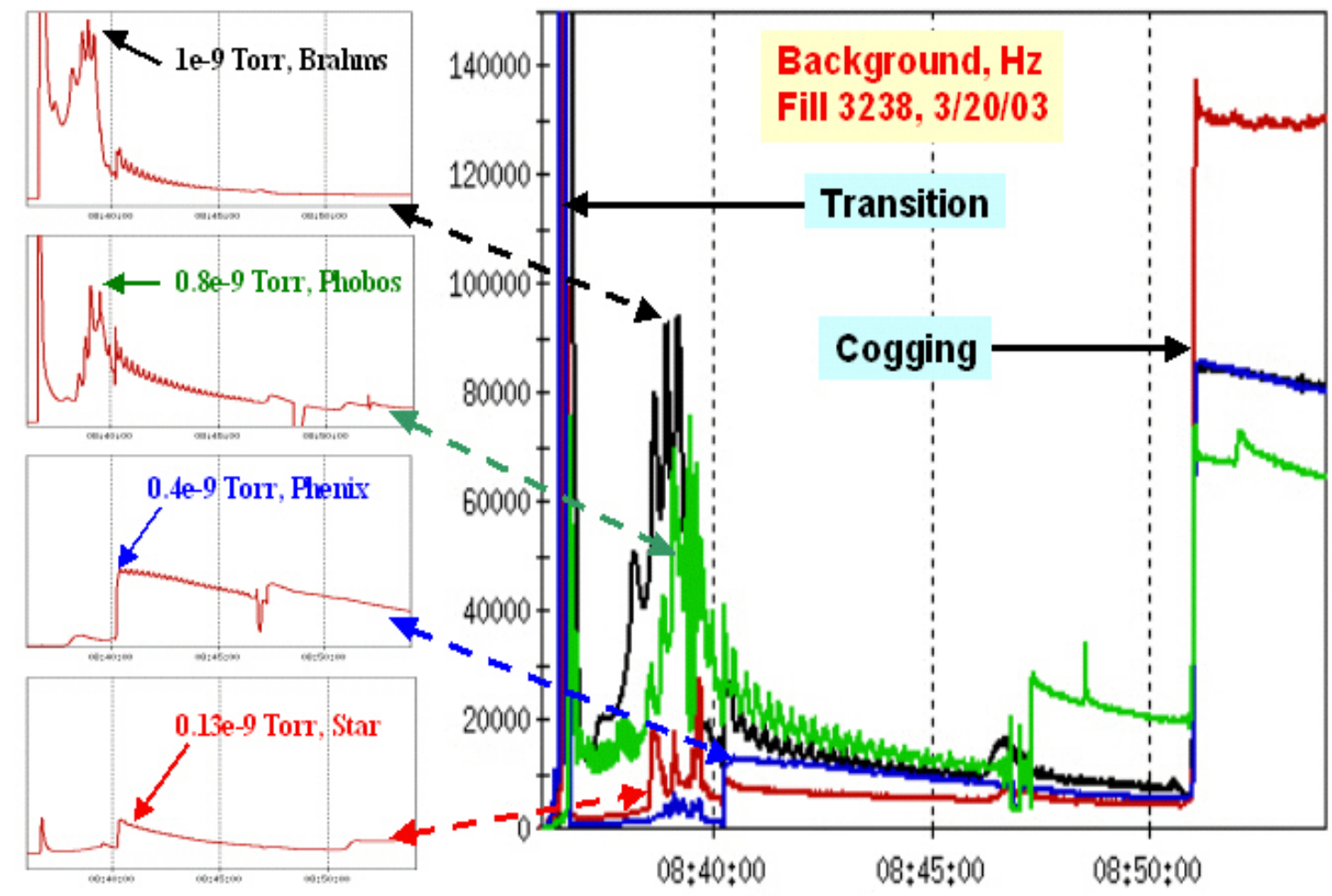

Fig.1: Background before and after beam cogging, and pressure rise at IRs.

Fig.1 shows a typical case for the beam-gas and beam-beam created background. Before the beam cogging, the background is dominated by the beam-gas, which is clearly coincided with the pressure rise. For Brahms, with peak pressure rise of $10^{-9}$ Torr, the background rate is about 90 $\mathrm{kHz}$. Also, for Phobos, Phenix, and Star, pressure rises of $0.8 \times 10^{-9}$ Torr, $0.4 \times 10^{-9}$ Torr, and $0.13 \times 10^{-9}$ Torr correspond background rates of $75 \mathrm{kHz}, 15 \mathrm{kHz}$, and $10 \mathrm{kHz}$, respectively.

Convention 11 indicates that at $\mathrm{P}=10^{-9}$ Torr, the $\mathrm{d}$-Au beam-gas created background is $\mathrm{Bk}_{\mathrm{d}-\mathrm{Au}, \mathrm{gas}}=78 \mathrm{kHz}$, which is approximately agreeable with the ones shown in Fig.1. Note that the beam intensity of Fill 3238 was $\mathrm{N}_{\mathrm{Au}}=35 \times 10^{9}$ ions, and $\mathrm{N}_{\mathrm{d}}=58 \times 10^{11}$ ions, close to the convention, i.e., $\mathrm{N}_{\mathrm{Au}}=40 \times 10^{9}$ ions, and $\mathrm{N}_{\mathrm{d}}=56 \times 10^{11}$ ions.

Since the beam-gas induced background has been significantly reduced at the time of beam cogging, the jump of background rate there is mainly due to the beam-beam collision. In Convention 10, the d-Au beam-beam background is estimated to be $107 \mathrm{kHz}$. Both Star and Phenix have $\beta^{*}=2 \mathrm{~m}$, therefore, the observed background at Phenix seems a little lower, and at Star, it is a little higher. Phobos and Brahms have $\beta^{*}=3 \mathrm{~m}$, therefore the observed background rate looks reasonable. In general, observation is approximately agreeable with the parameters derived from the convention. 


\section{Beam-gas dominated case}

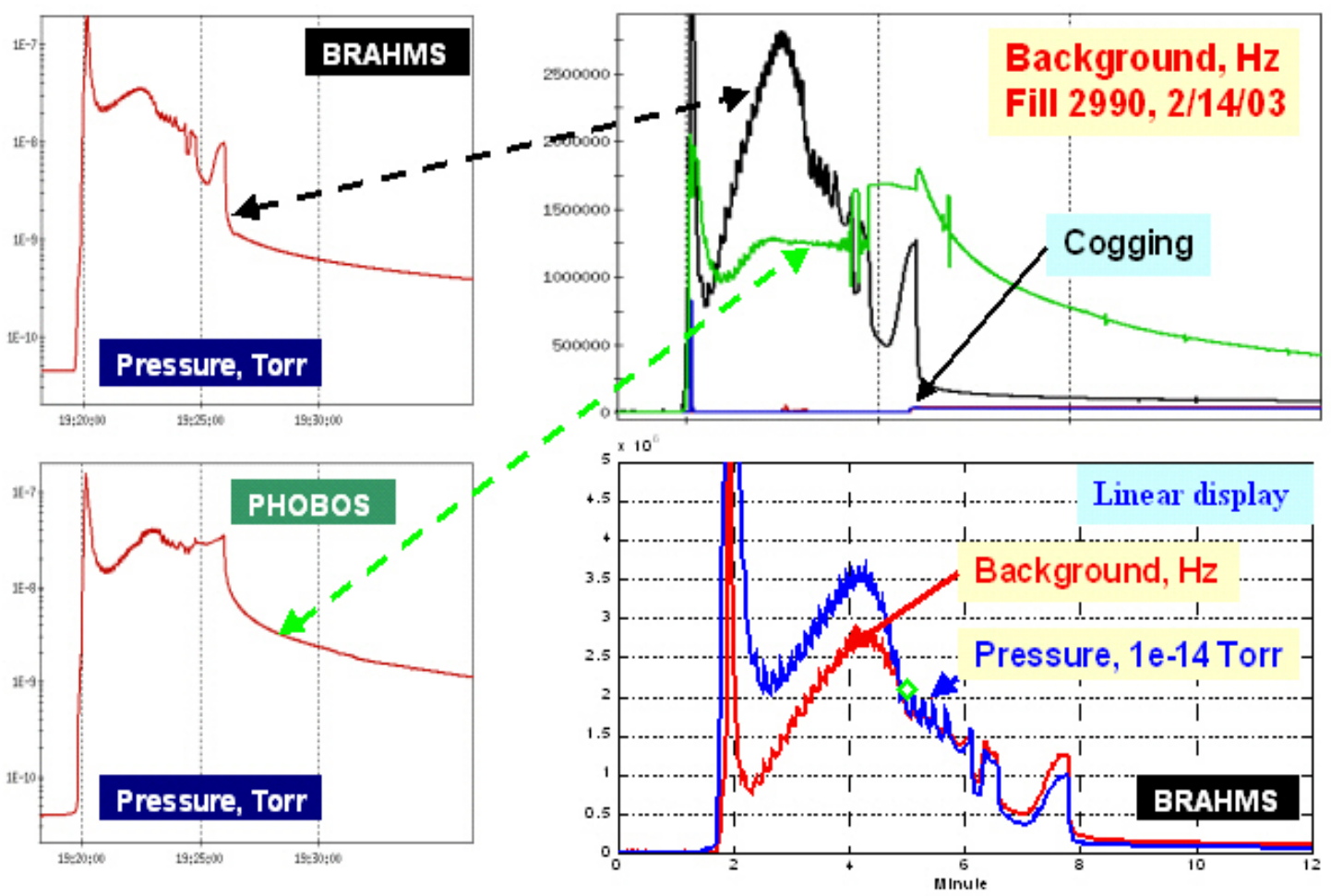

Fig.2: Beam-gas background, pressure rise, and linear display of both for Brahms

Fig.2 shows a case that the background is dominated by the beam-gas. Note that the background of Brahms and Phobos is very high, at 2 to $3 \mathrm{MHz}$, the relatively small beam-beam created background of Star and Phenix, about $50 \mathrm{kHz}$, are barely identifiable at cogging in Fig. 2 .

Coincidence of the background with respect to the pressure rise at the Brahms is clearly shown from the linear display in Fig.2. Also, the peak pressure rise there is $3 \times 10^{-8}$ Torr after the transition, and the corresponding peak background of Brahms is about $2.7 \mathrm{MHz}$, approximately $90 \mathrm{kHz}$ background per $10^{-9}$ Torr of pressure rise.

The very high pressure rise is due to the high total beam intensity. In Fill 2990, the gold beam intensity was $47 \times 10^{9}$ ions, and the deuteron beam intensity was $66 \times 10^{11}$ ions, which was one of the highest intensity fill in the run.

\section{Background review of d-Au run}

\section{ZDC coincidence}

The ZDCcoin history of all experiments in d-Au run is shown in Fig.3, with 67 high intensity fills starting from 1/17/03. There were 4 events during the run, which affected ZDCcoin, theses are: 

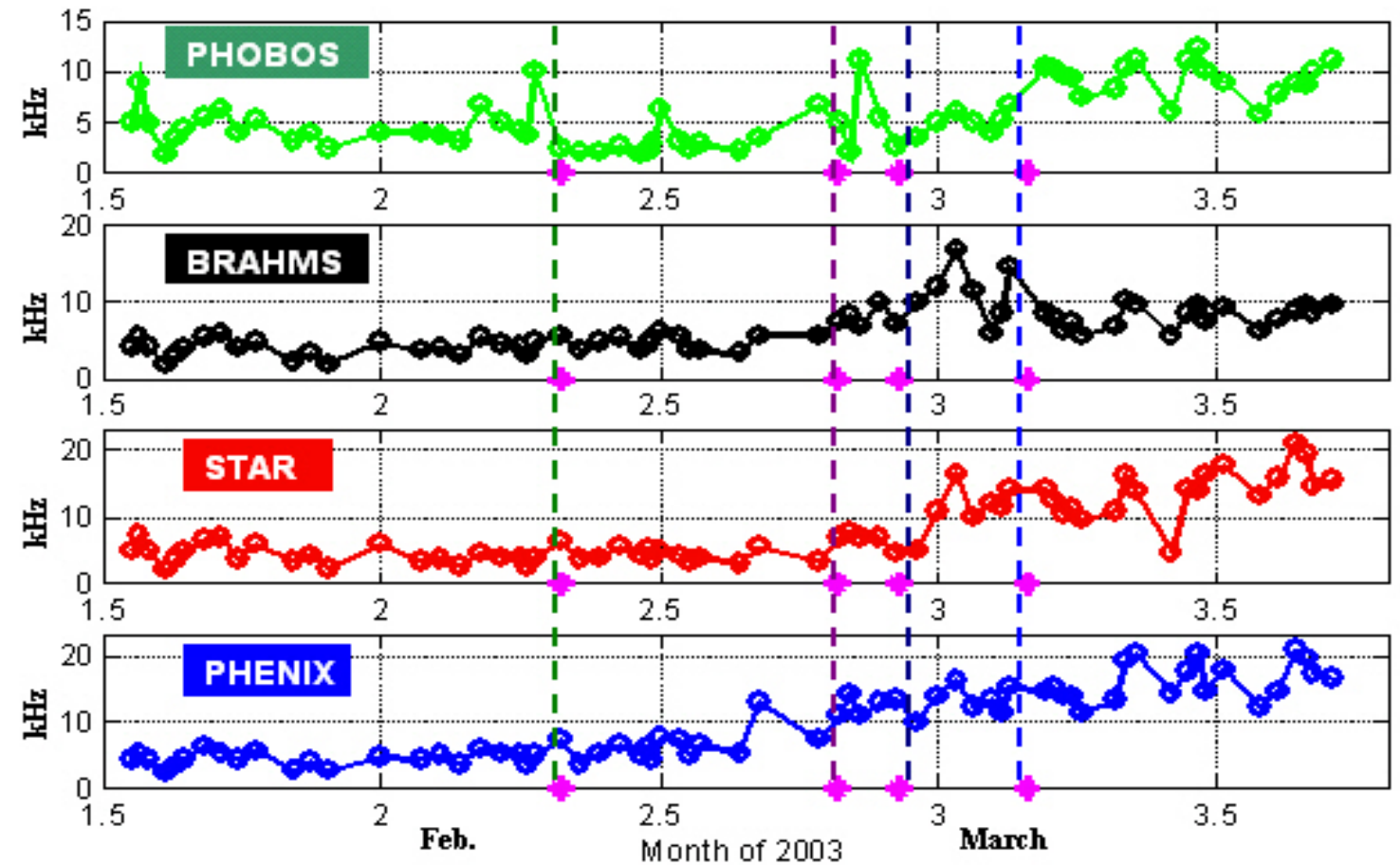

2/9: dAu3, Phobos betastar from $2 \mathrm{~m}$ to $4 \mathrm{~m}$, rest is $2 \mathrm{~m}$.

2/23: Coherence resolved, bhe beam emittance reduced.

2/26: 110 bumch to 55 bunch, total intensity dropped to $1 / 2$. 3/5: dAu4, Brahms and Phobos betastar to $3 \mathrm{~m}$, rest is $2 \mathrm{~m}$.

Fig.3: ZDCcoin history in d-Au run

1. 2/9/03. dAu2 optics was switched to dAu3. $\beta^{*}$ at Phobos was changed from $2 \mathrm{~m}$ to $4 \mathrm{~m}$, and others remained at $2 \mathrm{~m}$. The reduction of ZDCcoin of Phobos can be identified in Fig.3. Prior to the switch, the background and radiation level at Phobos were high, presumably due to the high total beam intensity and tight aperture there. Abort kicker misfiring and loss monitor permit pulling also frequently occurred. The optics switch helped, but not enough.

2. $\mathbf{2} / \mathbf{2 3} / \mathbf{0 3}$. Blue beam coherence after transition was eliminated by raising chromaticity around the transition. The blue beam emittance was reduced by about a factor of 2 at the collision. It can be observed in Fig.3 that the Phenix ZDCcoin was jumped at that point, about doubled.

3. $\mathbf{2 / 2 6 / 0 3}$. The background at Phobos was found to be proportional to the pressure rise at IR10, pressure rise was found to be, almost quasi-exponentially, proportional to the total beam intensity [9]. To reduce the total beam intensity, 110-bunch was switched to 55-bunch mode. Pressure rise was significantly reduced, and the background was improved.

4. $\mathbf{3} / \mathbf{5} / \mathbf{0 3}$. Brahms still had high background (this might be actually caused by beam loss there), $\mathrm{dAu} 4$ optics applied, with the $\beta^{*}$ at Brahms increased from $2 \mathrm{~m}$ to $3 \mathrm{~m}$, while Phobos reduced from $4 \mathrm{~m}$ to $3 \mathrm{~m}$. Star and Phenix remained unchanged at $2 \mathrm{~m}$.

We note that in this figure, and in this report as well, the beam intensity was taken at the transition, the background, ZDCcoin, and the associated pressure rise are all taken at 30 minutes after the transition, if not otherwise stated. 


\section{Total and bunch intensity}

The history of total beam intensity (in the unit of $10^{9}$ ions for gold, and $10^{11}$ ions for deuteron) and bunch intensity is shown in Fig. 4 .

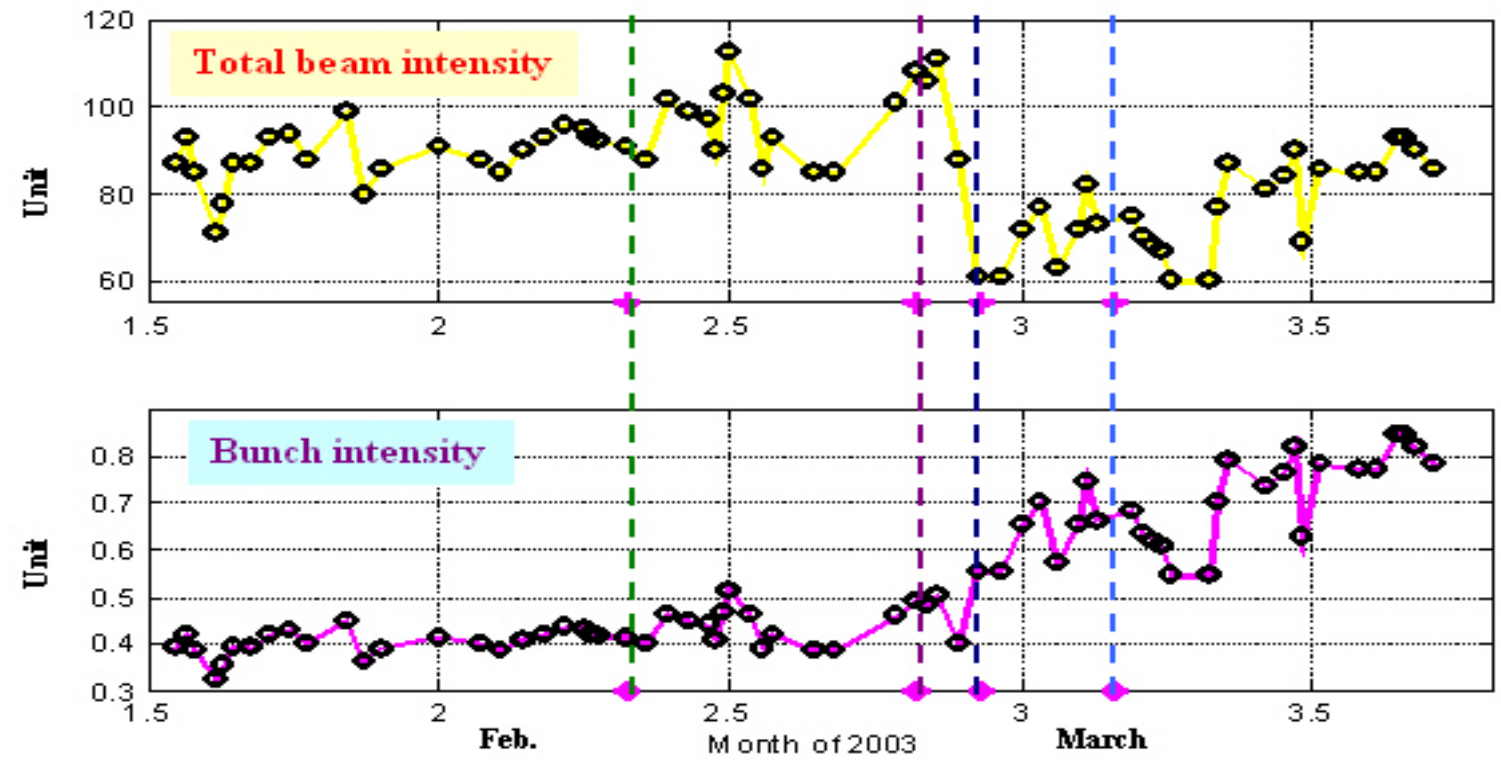

Fig.4. History of total beam intensity and bunch intensity

The large drop of the total intensity at the switch from 110-bunch to 56-bunch mode helped to reduce the high pressure rise, hence the beam-gas background. Meanwhile, the bunch intensity was closely related to the beam-beam background, which is shown in Fig.5.
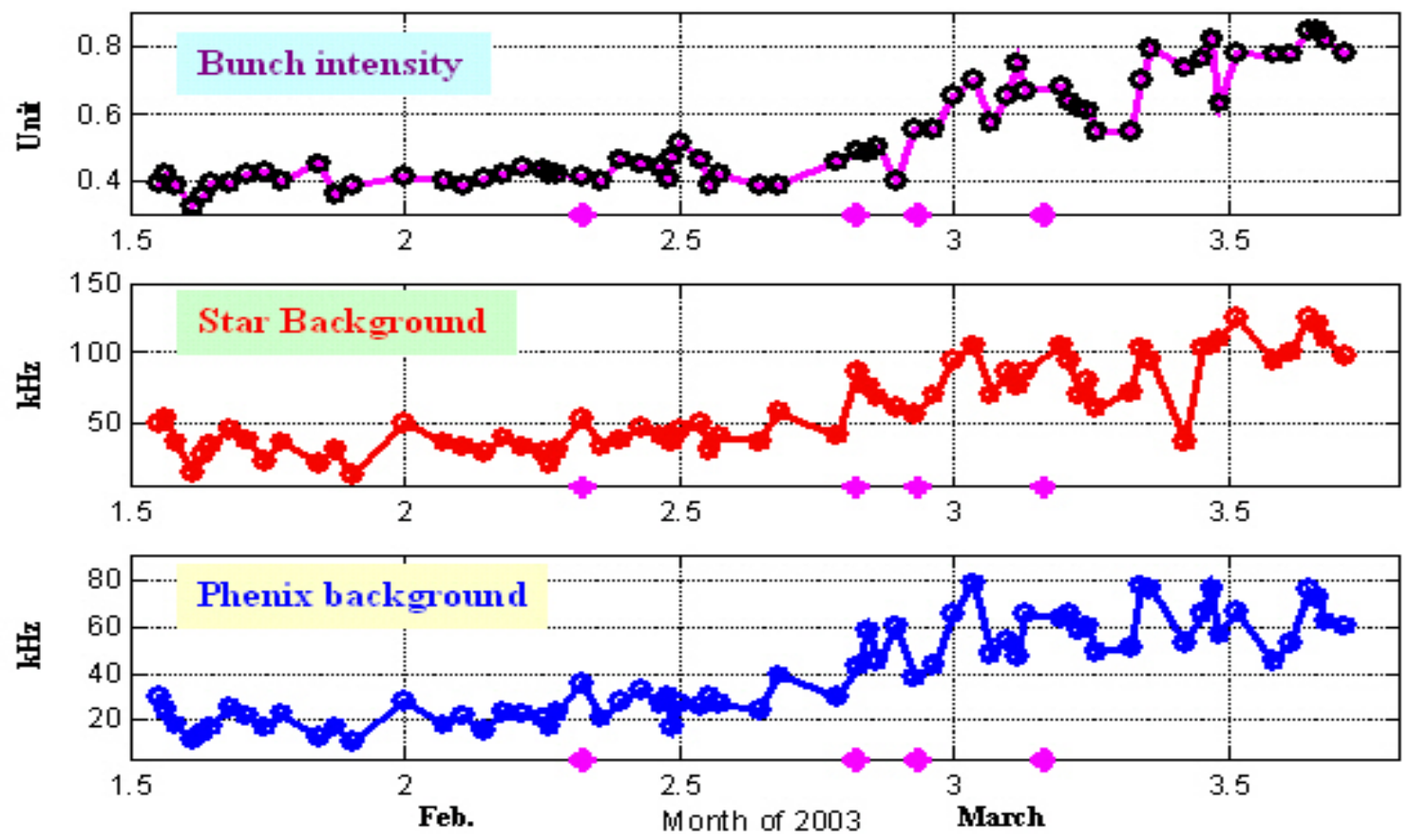

Fig.5. Background at Star and Phenix increases with the bunch intensity 


\section{Ratio of background/ZDCcoin}

The most relevant parameter perhaps is the ratio of background/ZDCcoin, rather than the background itself. In Fig.6, the history of this ratio is shown.
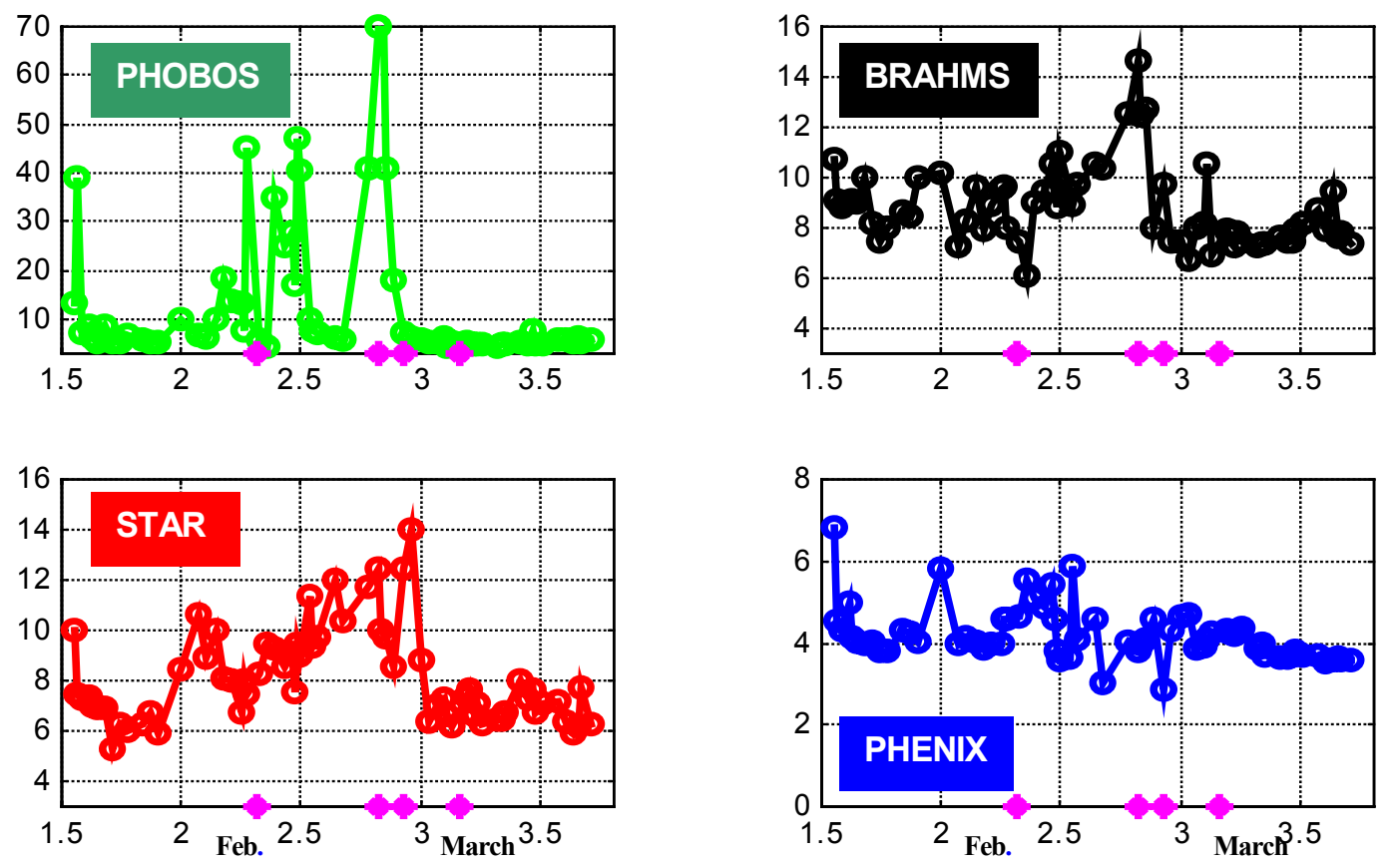

Fig.6. History of background/ZDCcoin ratio

At the later run, it is apparent that all experiments' background/ZDCcoin ratio was improved. The improvement of Phobos is most significant, whereas the one of Phenix is much less.

In the next section, the background problem for each experiment will be discussed.

\section{Background for Phobos, Brahms, Star and Phenix}

\section{Phobos}

The Phobos background/ZDCcoin ratio in two different scales, and the total beam intensity are shown in Fig.7.

The background/ZDCcoin is closely related to the total beam intensity. Below the total beam intensity of 90 units, this ratio is about 5. If the intensity is above 100 units, the background/ ZDCcoin ratio rises rapidly.

The transition pressures rises at several interaction regions are increased with the total beam intensity, and this pressure rise seems responsible to the Phobos background.

In Fig.8, it is shown that the transition pressure rises at IR2, IR10, and IR12 are almost quasiexponentially proportional to the total beam intensity. In Fig.9, it is shown that the background of Phobos is proportional to the pressure rise at IR10. 


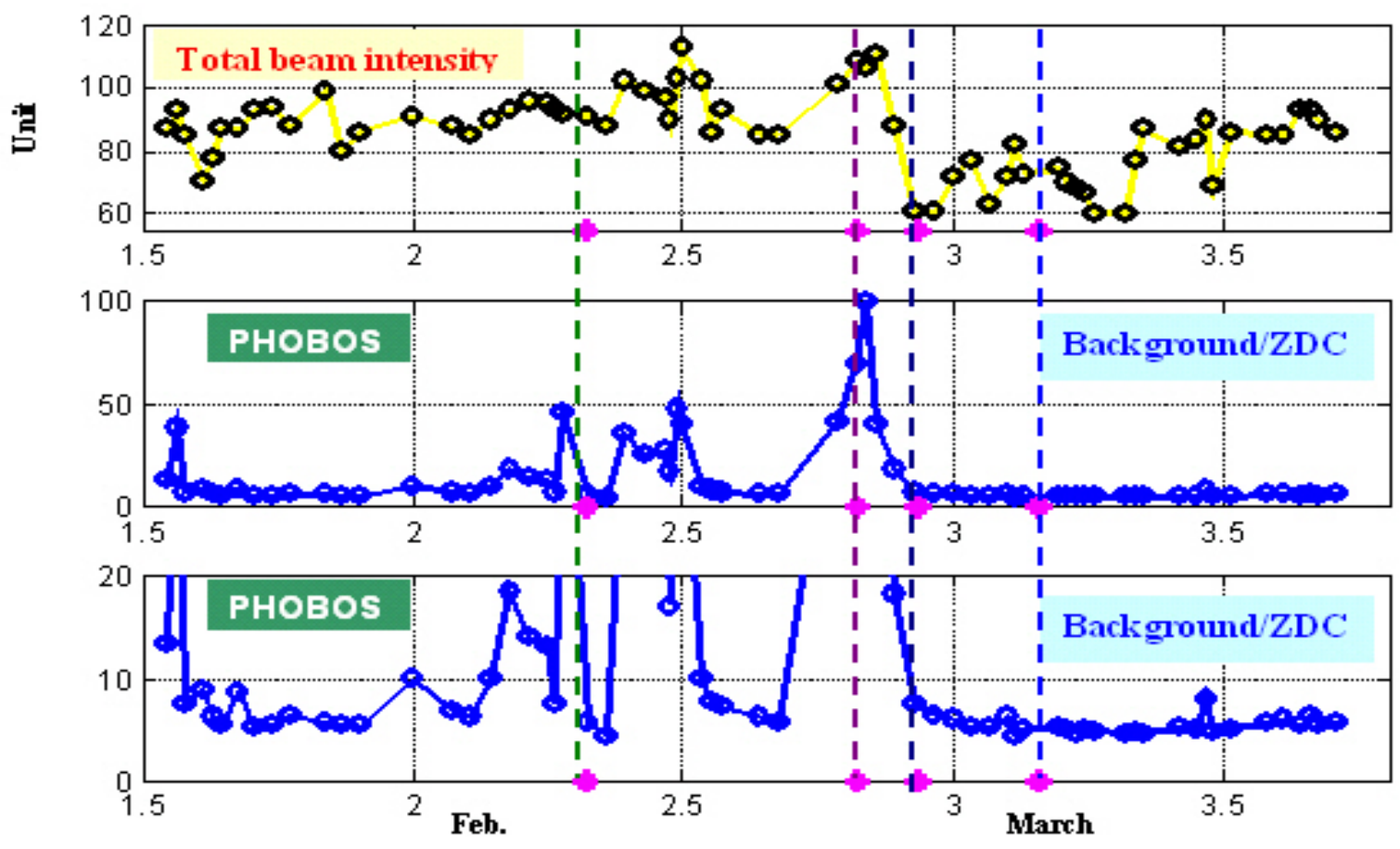

Fig7. Total intensity and Phobos background/ZDCcoin

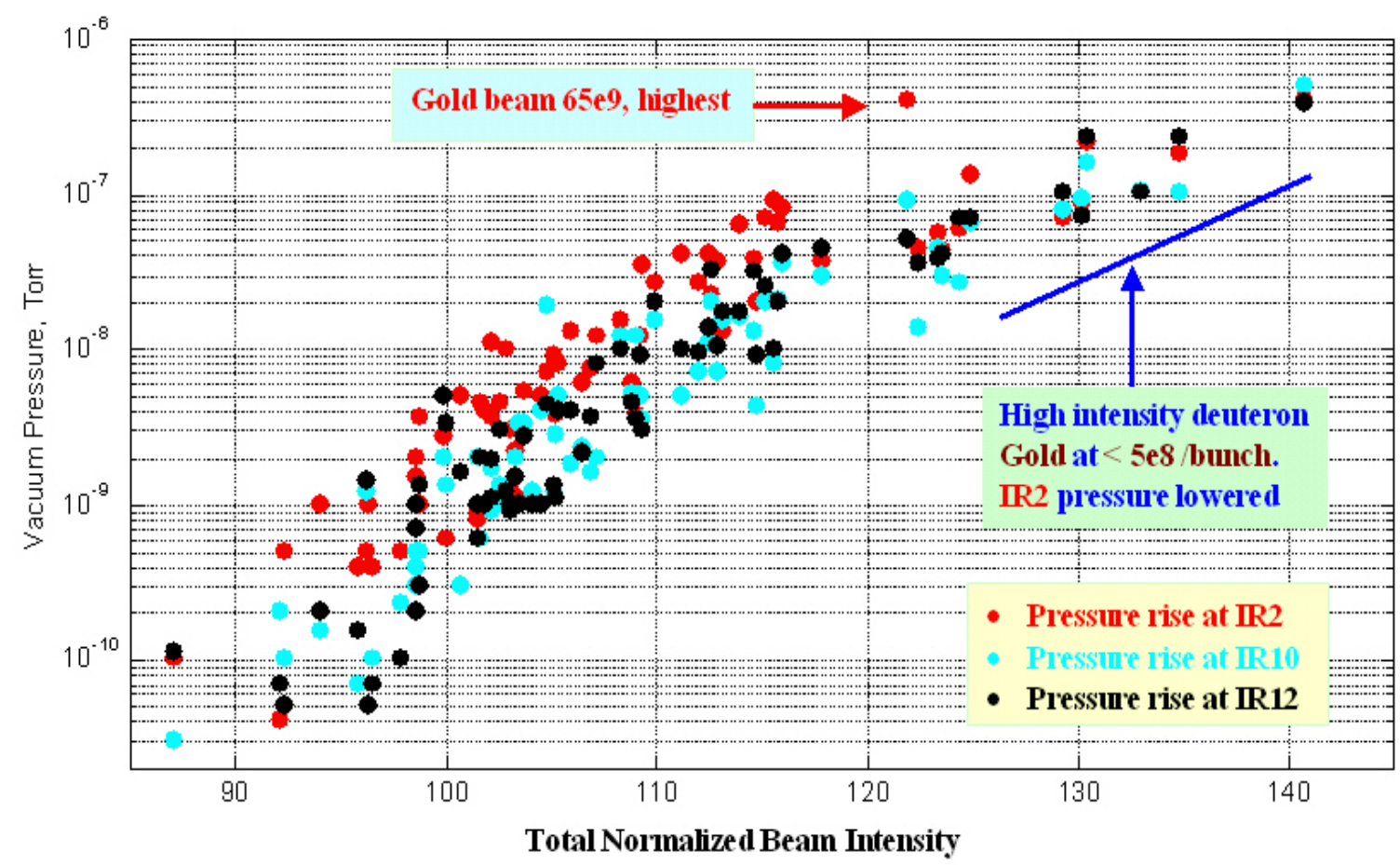

Fig.8. Transition pressure rise at IR2, IR10, and IR12 vs. total beam intensity

As a brief summary, the high total beam intensity induced pressure rise at transition seems to be primarily responsible to the Phobos background problem. The fact that the collimation was unable to reduce the Phobos background indicates that the beam loss/scraping is not the dominant factor there, agreeable with this conclusion. 


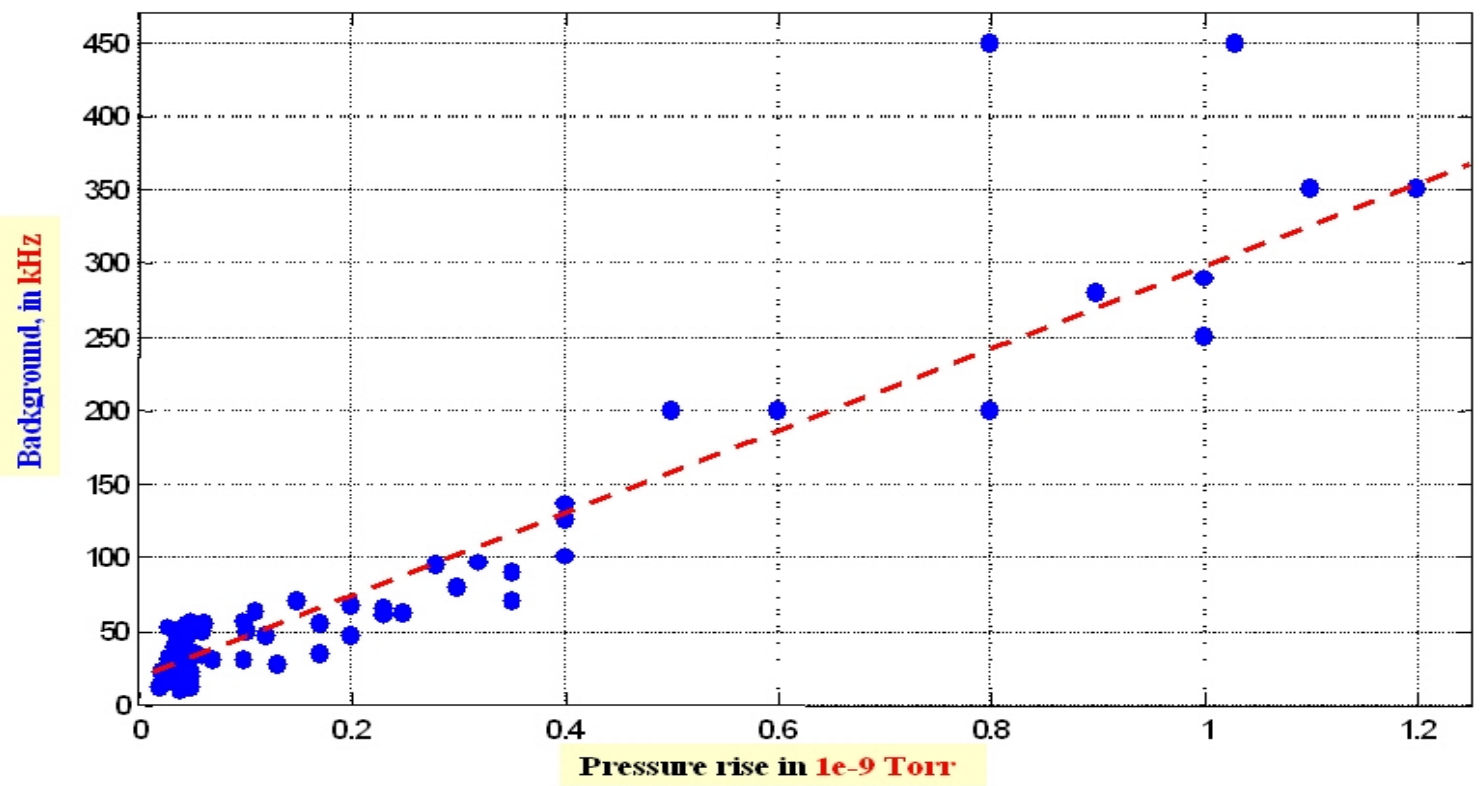

Fig.9. Phobos background vs. pressure rise

\section{Brahms}

In about 3 weeks during the d-Au run, from $2 / 13$ to $3 / 5$, the Brahms background was often, but not always, affected by beam loss. In Fig.10, a typical beam loss induced Brahms background, in an hour at early store, and the related loss monitor observation, are shown.

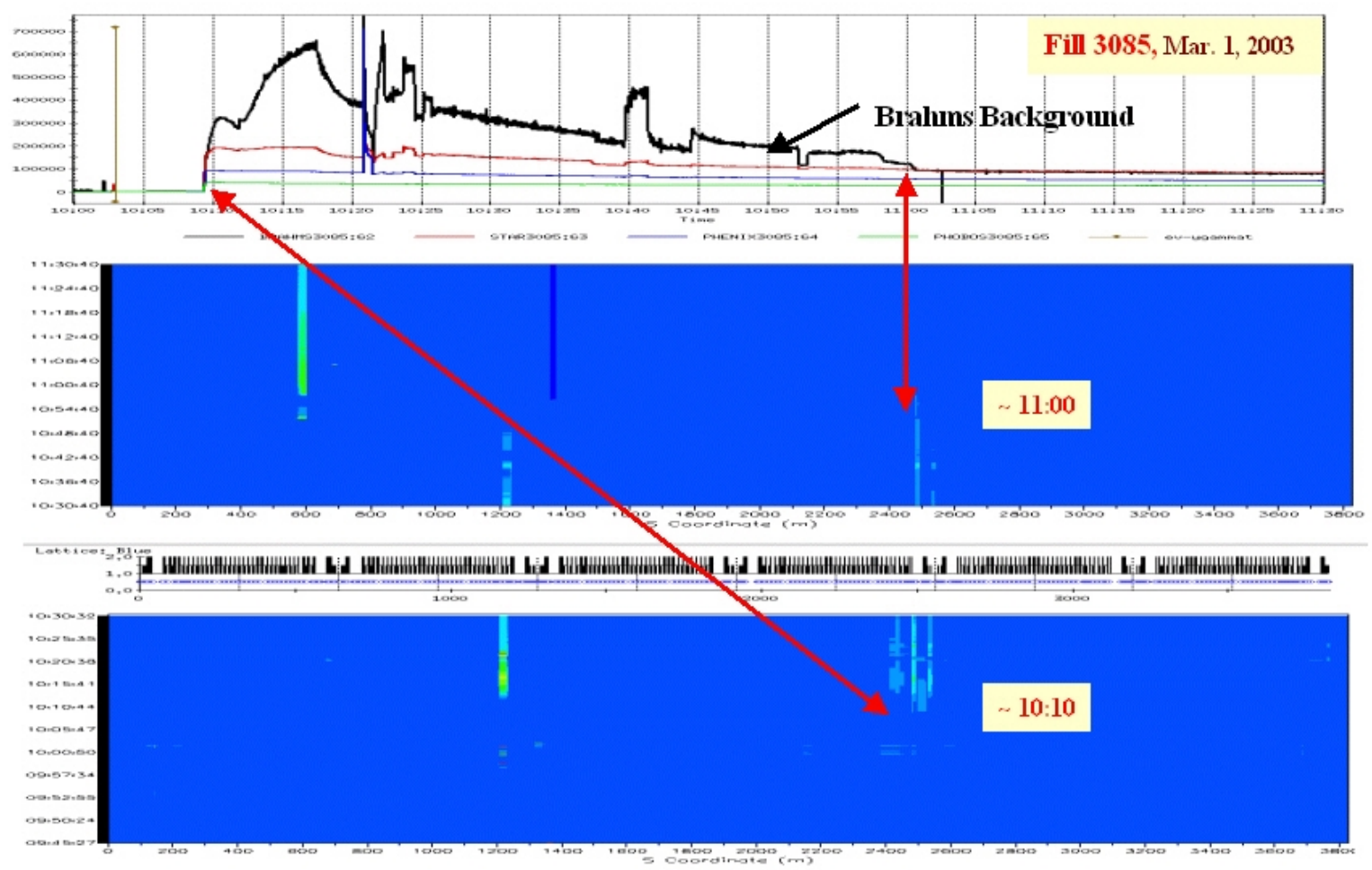

Fig.10. Brahms background and the related beam loss observed by loss monitor 
To look into the Brahms background for effects other than the beam loss, two sets of background data were identified. One is the background observed as is, and another is the one excluding the beam loss effect. This is illustrated in Fig.11.

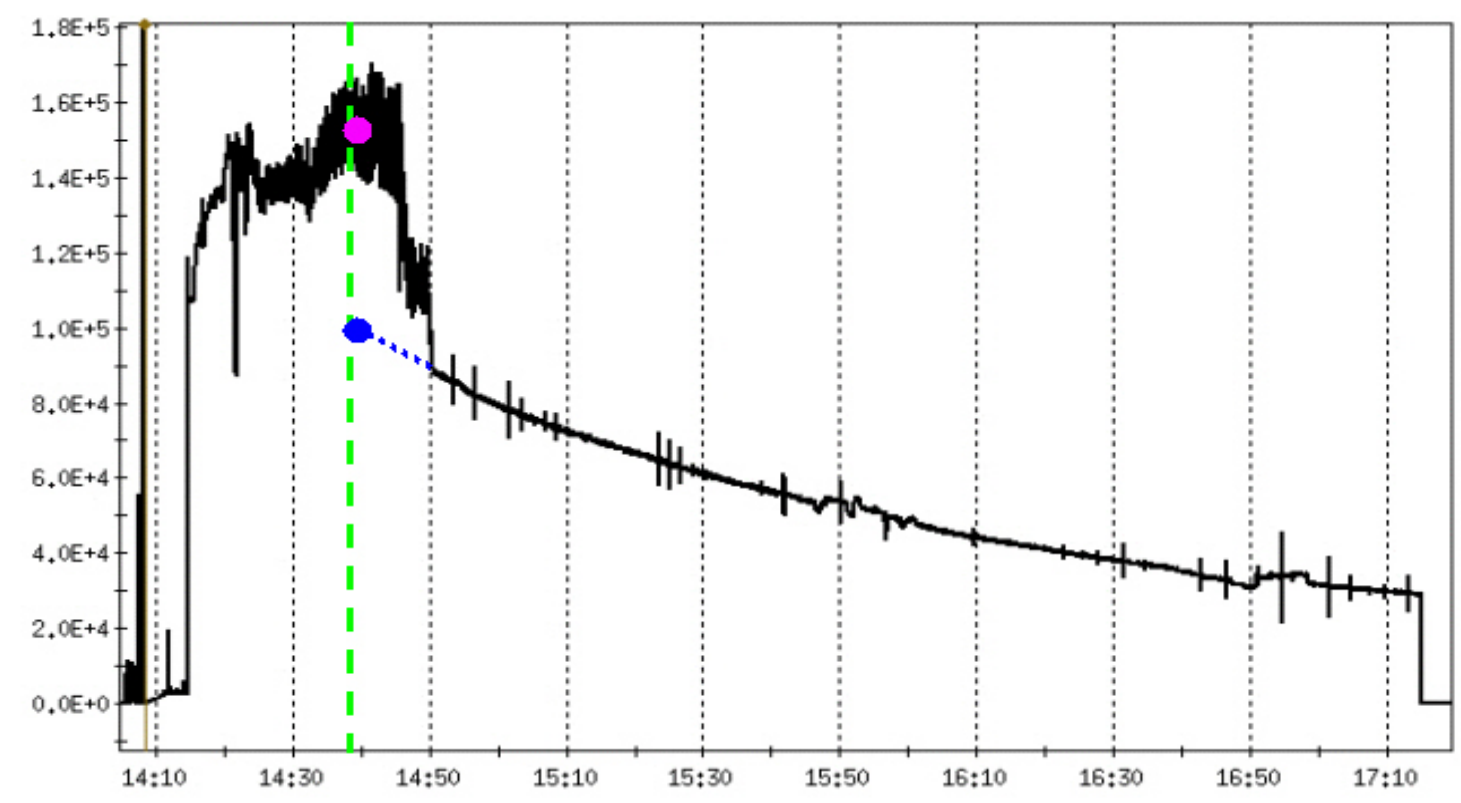

Fig.11. Magenta dot: the background, blue one: excluding beam loss effect

In Fig.12, the total beam intensity and these two sets of data are shown.

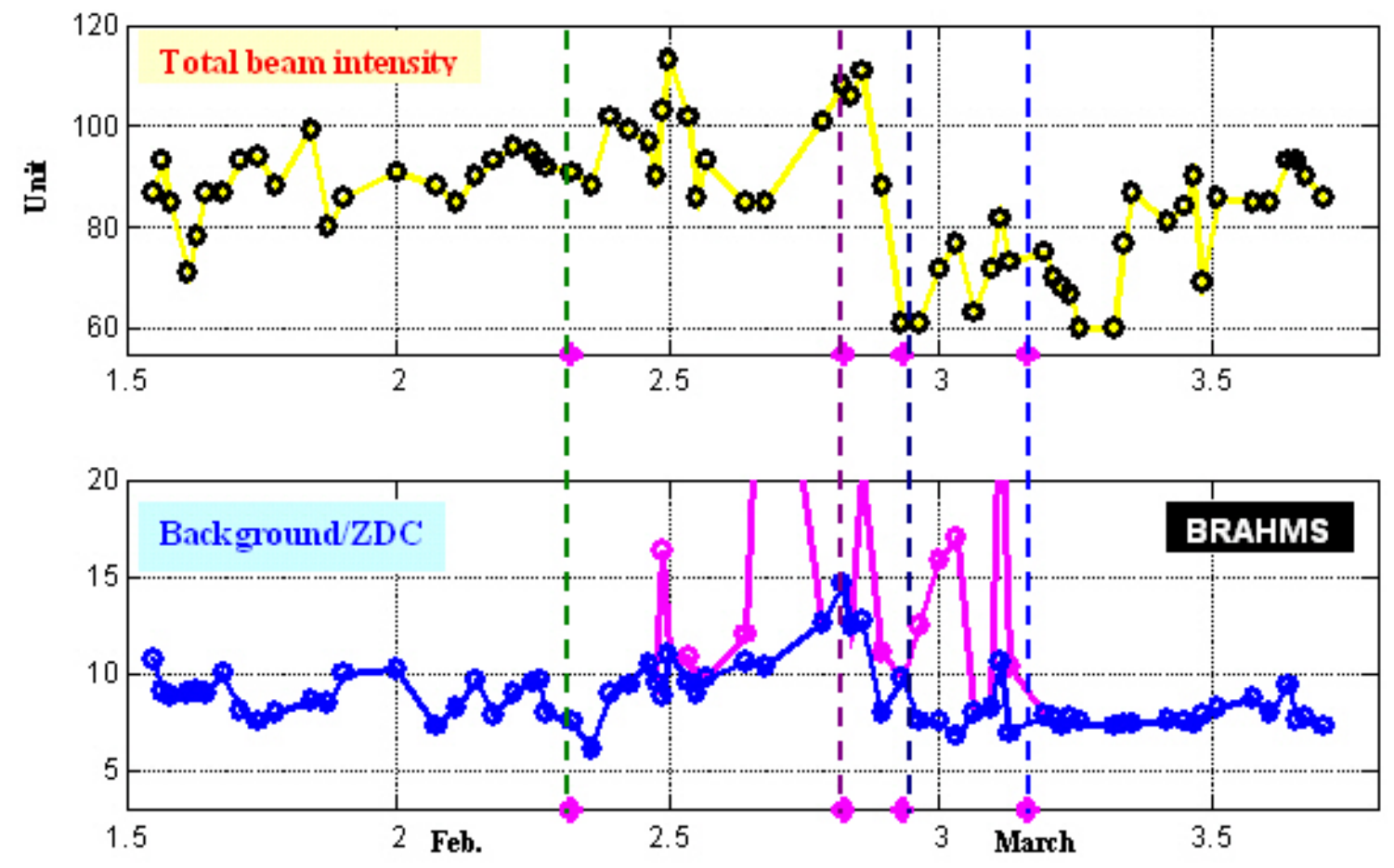

Fig.12. Total intensity and Brahms background/ZDCcoin 
The Brahms background was significantly improved after 3/5/03, when the $\beta^{*}$ at the Brahms was changed from $2 \mathrm{~m}$ to $3 \mathrm{~m}$. The reduced beam transverse size at the triplet with $\beta^{*}=3 \mathrm{~m}$ certainly helped to improve the beam scraping, and hence the background. However, there are indications that the beam loss at the Brahms may not be solely due to the optics limitation.

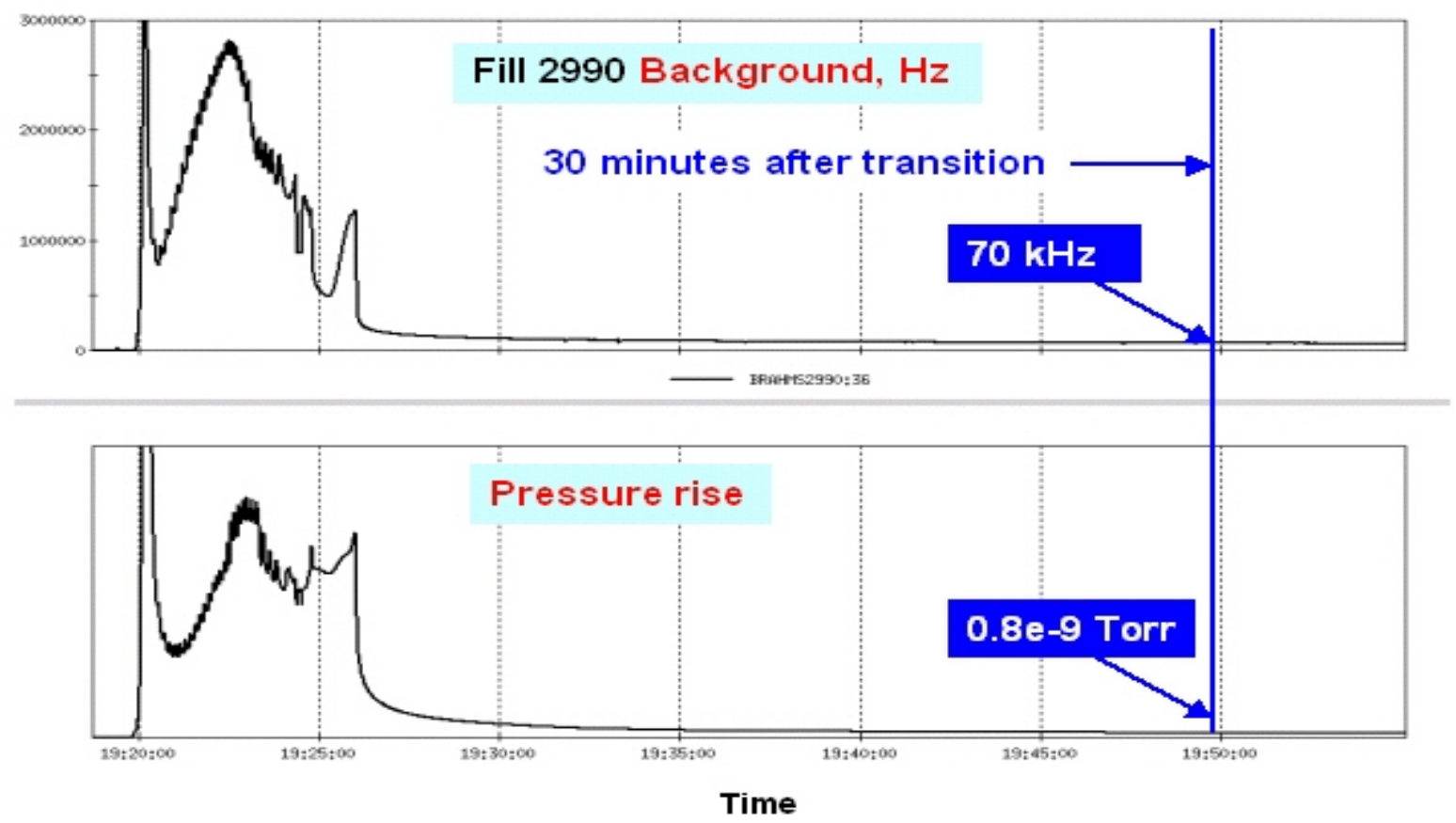

Fig.13. Background and pressure rise at Brahms, Fill 2990

In Fig.13, the background and pressure rise at Brahms are shown for Fill $2990\left(\beta^{*}=2 \mathrm{~m}\right.$ then). In this case, no significant beam loss was observed. The large background between 19:20 (beam transition) to 19:26 are due to the high pressure rise, which is also shown. At 30 minutes after the transition, the background was about $70 \mathrm{kHz}$, which is compared with the ZDCcoin of $6.2 \mathrm{kHz}$. The background/ZDCcoin ratio is not large. Excluding the background from the pressure rise, which was rather high at $0.8 \times 10^{-9}$ Torr, this ratio was actually even lower. This indicates that the beam loss at the Brahms with $\beta^{*}=2 \mathrm{~m}$ could be minimized, or eliminated.

Back to Fig.12. Excluding the beam loss factor, the decrease of background/ZDCcoin ratio at the end of February shows that the Brahms background could be affected by the total beam intensity through the pressure rise. However, the presumably beam-gas effect at the Brahms is less significant, compared with the Phobos.

\section{Star}

The total beam intensity, ratio of background/ZDCcoin, and ZDCcoin of Star are shown in Fig.14.

The improvement of the background/ZDCcoin ratio at the end of February is clearly visible, indicating that the Star background was also affected by the high total beam intensity. However, the pattern is not as consistent and dramatic as that of Phobos. During that period of the operation, the beam loss at the Star often took place, which might play a role in background. 


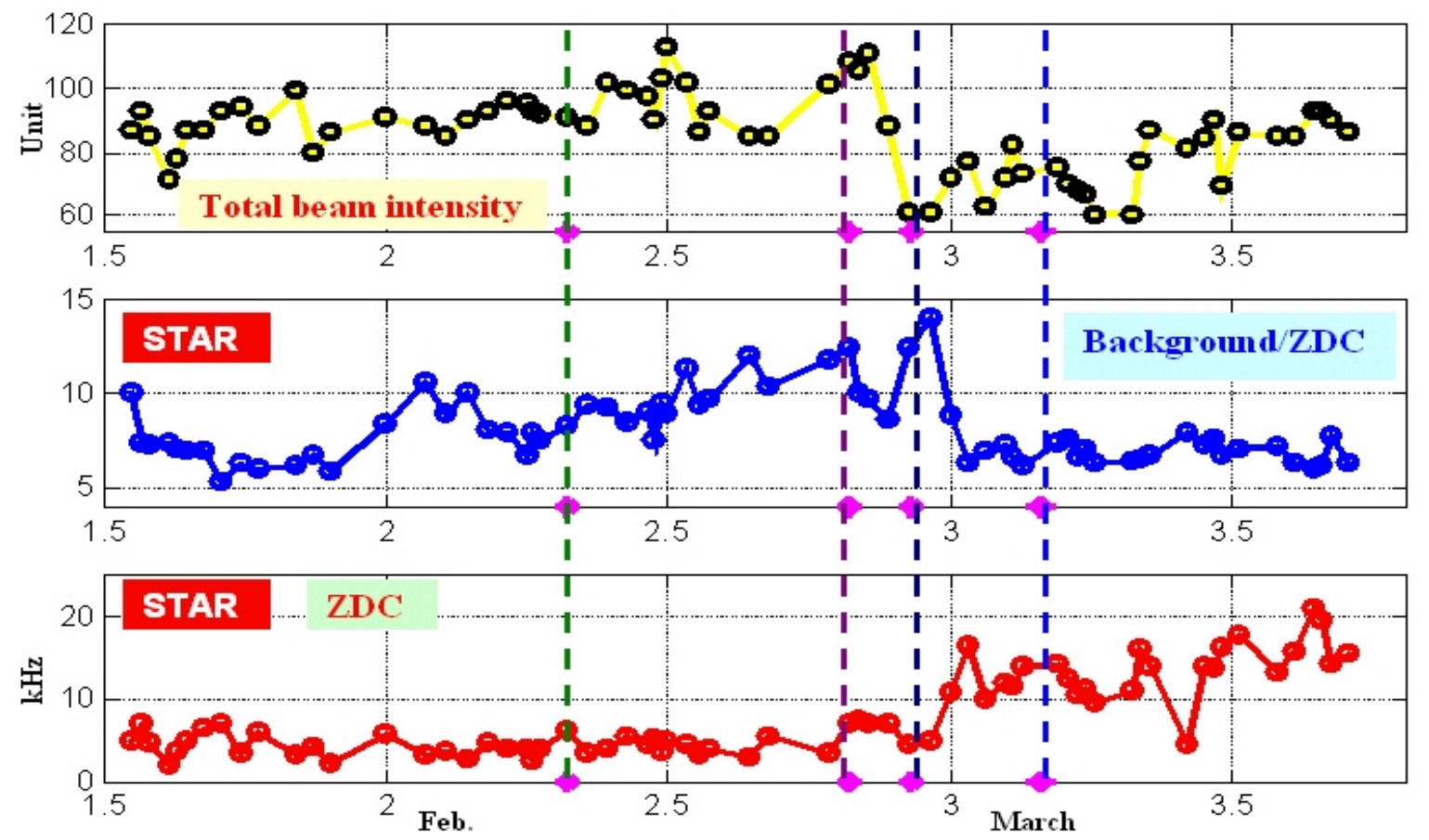

Fig.14. Total intensity, background/ZDCcoin and ZDCcoin at Star

\section{Phenix}

In Fig.15, the total beam intensity, the ratio of background/ZDCcoin, and ZDCcoin of Phenix are shown.
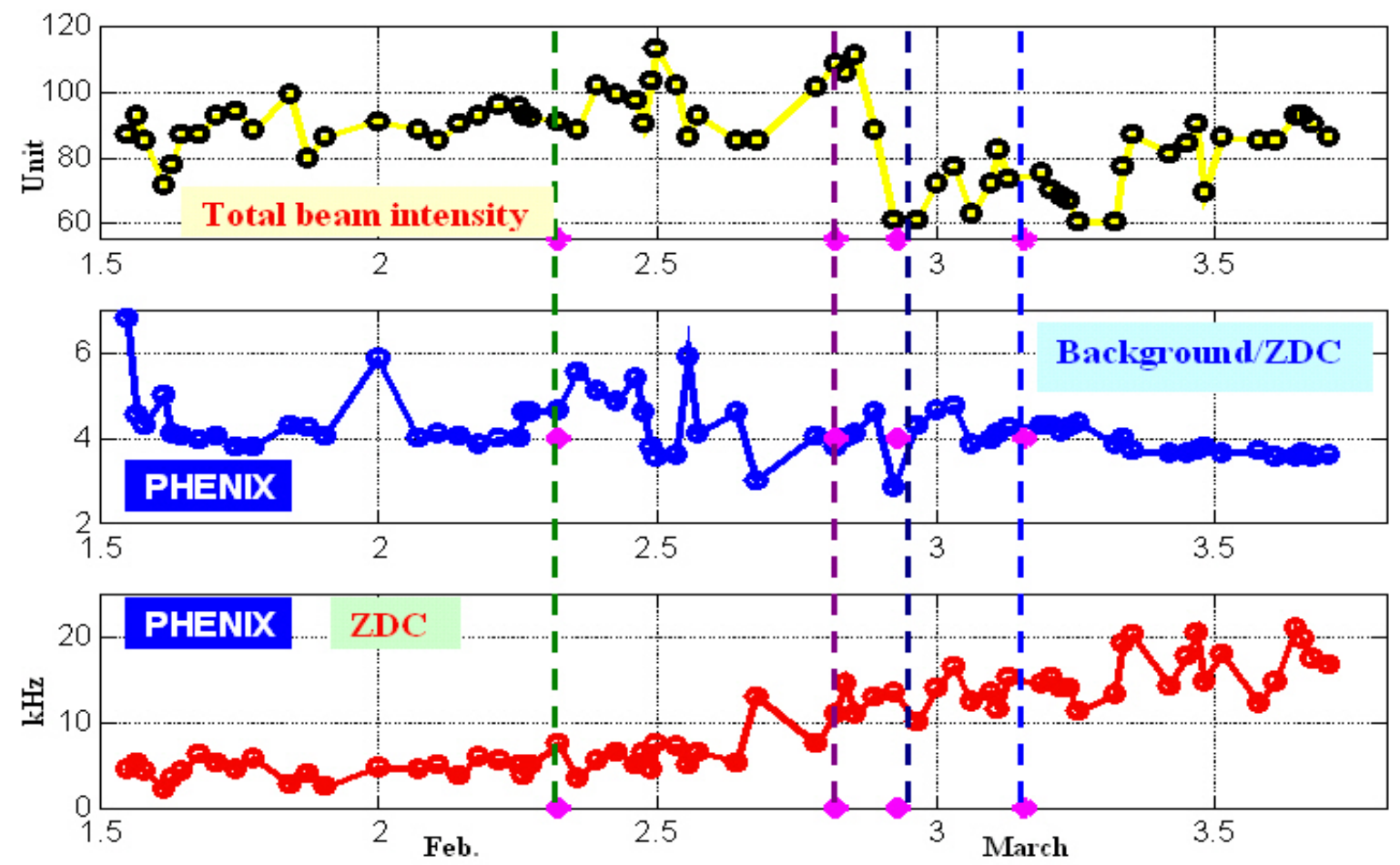

Fig.15. Total intensity, background/ZDCcoin, and ZDCcoin at Phenix 
As the beam coherence (instability) resolved at $2 / 23 / 03$ by raising the chromaticity at the transition for deuteron beam, the Phenix ZDCcoin was almost doubled, presumably due to the smaller beam emittance at the collision. There is no clear indication of the influence of the total beam intensity, or anything else.

\section{Beam-gas factor in background}

In Fig. 16, beam intensity, and the pressure rise at all experiments, for the fills from the time period of $2 / 22$ to $2 / 28 / 03$, are shown.

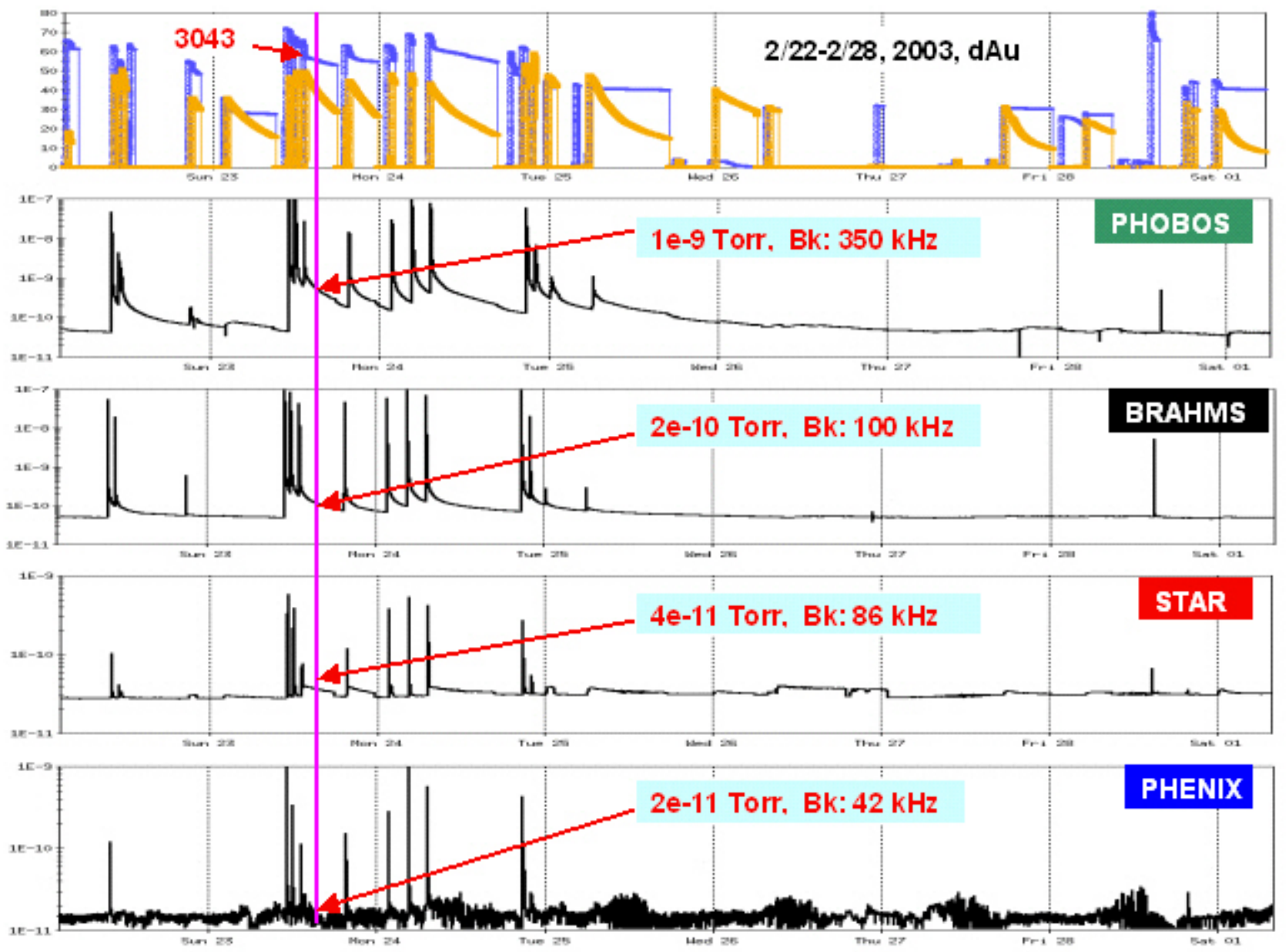

Fig.16. Beam intensity, and pressure rise at all experiments, from $2 / 22$ to $2 / 28 / 03$

Peaks of the pressure rise are at the beam transition, these are in the order of $10^{-7}$ Torr for Phobos and Brahms, and in $10^{-9}$ Torr for Star and Phenix. The pressure rises were disappeared after 2/26/03, when the 110-bunch mode was changed to 56-bunch.

The pressure rise at 30 minutes after the transition of Fill 3043, and the background at the same time are shown in Fig.16. Note that at the time, the background consisted by both beam-gas and beam-beam. The background at the Phenix was mainly due to the beam-beam, whereas at the Phobos, the beam-gas background was clearly dominant. For Brahms and Star, it seems that the beam-gas may have contributed to the background. 
The long pressure decay time at the Phobos, approximately 15 hours from $10^{-9}$ Torr to $10^{-10}$ Torr, caused the pressure accumulation, which contributed significantly to the background.

At the Phenix, the very steep decrease of the transition pressure rise to the very low quiescent level looks helped the background, but the smaller magnitude of the transition pressure rise is probably also relevant in the fast pressure decay.

In Fig.17, the pressure rise at the Phobos, for Fills 3046, 3048, and 3049, is shown, where the pressure accumulation can be observed for the gauges of $\mathrm{g} 9$ and g10 pwx, which are located 7.6 $\mathrm{m}$ from the IP, at sections 9 and 10, respectively. The gauges at DX-DO, close to DX magnets, however, show very little pressure accumulation. This indicates that the pressure rise at the Phobos, for other experiments as well, is a local problem. Therefore, pressure rise improvement at Q3 -Q4 straight sections may not help the beam-gas created background problem.

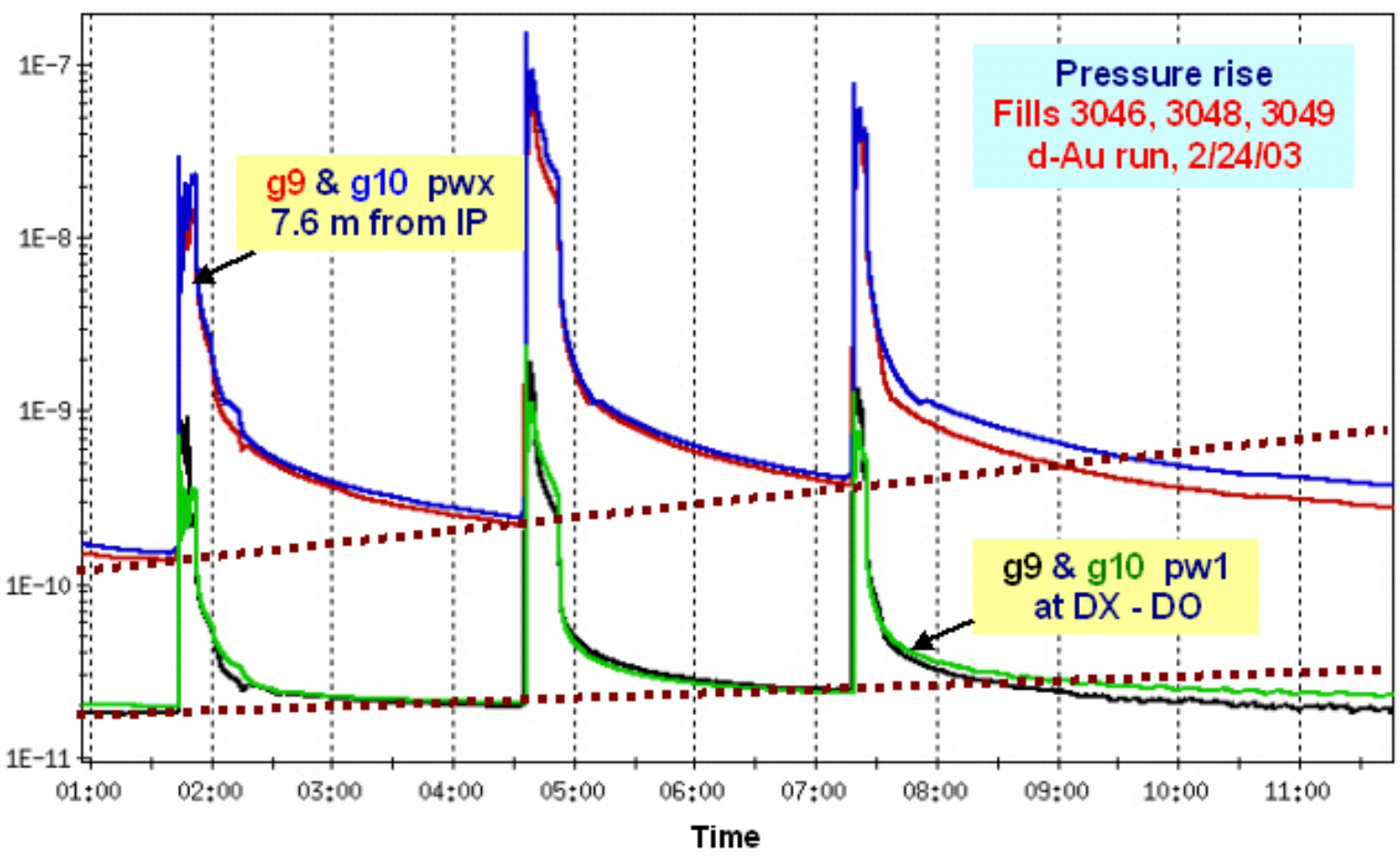

Fig.17. Comparison of pressure rise at g9,10 pwx and g9,10 pw1 for fills 3046,3048 and 3049

\section{Summary and discussion}

1. In this report, ZDC singles and ZDC coincident rate are used as the background and coincidence for all experiments. Discrepancy between the ZDC approach and others exists. For example, Brahms is using TOF(time of flight), and Phenix is using MuID(muon identifier) and $\mathrm{BBC}$ (beam beam counter) for collision event triggering. However, review and discussion have shown that the ZDC approach has indeed revealed useful information on the background problem.

2. Conventions of $\mathrm{Au}-\mathrm{Au}$ and $\mathrm{d}-\mathrm{Au}$ collisions in terms of the machine and beam parameter, vacuum condition, beam intensity, cross sections such as the nuclear collision, Coulomb dissociation, and beam-gas collision, have been proposed and checked for the past runs. 
Further examination is needed, especially in the coming Au-Au run. If proved applicable, then it can be used to analyze and predict, in a more general way, the experiment background problem. The d-Au hadronic interaction and gold beam gas interaction are not fully understood at the moment, which requires further investigation, and perhaps some beam study in the coming runs.

3. Background due to beam-beam, beam-gas, and beam-loss have been identified. The single Coulomb dissociation is believed a main contributor in the beam-beam collision created background. This type of background is in nature inherent, which leads to the minimum ratio of background/ ZDCcoin about 10 in Au-Au run, and about 4 in d-Au run. On the other hand, the beam-gas and beam-loss had generated much high background in the past $\mathrm{d}-\mathrm{Au}$ run. The relevant issues in reducing the backgrounds created by beam-gas and beam-loss include: the high total beam intensity induced transition pressure rise, possible remedy of the pressure rise using chamber coating and pumping, beam injection pattern, machine tuning, and the machine aperture limitations.

4. This approach is not very effective in identifying the beam-halo created background, which is treated separately from the beam-loss induced background. The beam-loss background refers to the cases where the corresponding beam loss can be identified from the beam loss monitor, and therefore can be minimized and/or eliminated by machine tuning. It remains to see if the ZDC approach can be applied to deal with beam-halo created background.

5. The Phobos background problem is clearly related to the pressure rise. High total beam intensity induced pressure rise at IR10, and the long pressure decay time there, are both responsible. Although the peak pressure rise at Brahms is almost comparable to the Phobos, better pressure decay there made large difference. As the result, the pressure rise factor in the Brahms background is much less significant than the Phobos. The Brahms, however, suffered beam loss induced background, for that larger $\beta^{*}$ and better machine tuning may help. Star may be affected by the pressure rise due to high intensity, and the background at the Phenix was not affected by either pressure rise and beam loss.

6. For the next Au-Au run, given the machine condition basically unchanged, the transition pressure rise may happen. With the bunch intensity of $\mathrm{N}_{\mathrm{Au} \text {,bh }}=10^{9}$ ions in 56-bunch mode, the total beam intensity will be 112 unit, which is higher than the $2001 \mathrm{Au}-\mathrm{Au}$ run of less than 80 unit. The magnitude of the pressure rise, however, will probably be lower that the d-Au run at 110-bunch mode. The collision rates shown in Table 1 present the understanding of the d$\mathrm{Au}$ and $\mathrm{Au}-\mathrm{Au}$ coincidence and background. According to Table 1, the ratio of beam-beam background/ZDCcoin will be around 10 . Given pressure rise of $\mathrm{P}=10^{-9}$ Torr, then the total background/ZDCcoin ratio will be about 17 . This way, the background may be of concern in the coming Au-Au run. However, the background problem will not be as serious as the past d$\mathrm{Au}$ run, where the background/ZDCcoin ratio of higher than 30 at the Phobos and Brahms had affected the machine operation.

\section{Acknowledgment}

I would like to thank D. Barton, D. Beavis, V. Cianciolo, W. Christie, A. Drees, J. Dunlop, W. Fischer, R. Fliller, H.C. Hseuh, W. MacKay, Y. Makdisi, R. Majka, R. Pak, K. Read, T. Roser, D. Trbojevic, S. White, K.Yip, and Z-B Xu, for information and helpful discussions. 


\section{References}

1. A. Drees, private communication.

2. H.C. Hseuh, private communication.

3. A.J. Baltz, C. Chasman, and S.N. White, NIM A 417, pp. 1-8, 1998.

4. A. Drees, Collision signals from experiments and signal/background ratios, RHIC Collision Strategy Review, Jan. 2000.

5. Evidence from $d+A$ u measurements from final state suppression of high $\mathrm{P}_{\mathrm{T}}$ hadron in $A u+A u$ collisions at RHIC, J. Adams, et al, for Star collaboration, accepted by Phys. Rev. Lett. on July 11, 2003

6. S.N. White, private communication.

7. T. Roser, RHIC Retreat 2002.

8. T. Satogata, RHIC Retreat 2003.

9. S.Y. Zhang, et al, RHIC pressure rise and electron cloud, PAC, 2003. 